\title{
LAS OPORTUNIDADES DE LA RENTA BÁSICA EN AMÉRICA LATINA FRENTE A LA PANDEMIA DEL COVID-19: VULNERABILIDAD SOCIAL Y BLOQUEOS ESTRUCTURALES
}

\section{BASIC INCOME OPPORTUNITIES IN LATIN AMERICA IN THE FACE OF THE COVID-19 PANDEMIC: SOCIAL VULNERABILITY AND STRUCTURAL BLOCKS}

\section{Fernando Filgueira}

Universidad de la República, Montevideo, Uruguay

fernando.filgueira@cienciassociales.edu.uy

\section{Rubén M. Lo Vuolo}

Centro Interdisciplinario para el Estudio de Políticas Públicas, Buenos Aires, Argentina ciepp@ciepp.org.ar

Palabras clave: Renta básica, América Latina, protección social, COVID-19.

Keywords: Basic income, Latin America, social protection, COVID-19.

Resumen: Frente a la pandemia del COVID19, los gobiernos de América Latina han tomado medidas que tienen y tendrán un profundo impacto económico y social. Si bien muchas de estas medidas mitigan parte del costo inmediato de la pandemia y de las estrategias epidemiológicas para su contención, las mismas pueden terminar potenciando las vulnerabilidades de la estructura social y los déficits de los sistemas de protección social. En este escenario se observa el crecimiento del debate de propuestas como el ingreso ciudadano o renta básica. Pese a que sería deseable su implementación, no hay muchas posibilidades en el corto plazo porque la misma depende de cambios en la percepción de las elites, de la inversión para superar los déficits estructurales en la provisión de bienes públicos y de la transformación de las concepciones que sostiene a las actuales políticas de transferencias monetarias focalizadas y condicionadas.

Abstract: Faced with the COVID19 pandemic, the Latin American governments have taken measures that have and will have a profound economic and social impact. While many of this measures might mitigate the socioeconomic impact of the pandemic and of the epidemiological strategies to contain its spread 
they might end up enhancing the vulnerabilities of the social structure and the deficits of the social protection systems. In this scenario, the debate on proposals such as citizen income or basic income is growing. Although its implementation would be desirable, there are not many possibilities in the short term because it depends on changes in the perception of elites, investment to overcome structural deficits in the provision of public goods and the transformation of conceptions that supports the current policies of targeted and conditional cash transfers.

\section{Introducción}

La pandemia del COVID19 generó en América Latina $(A L)$ una triple crisis combinada y asimétrica: sanitaria, económica y social (CEPAL, 2020a, CEPAL, 2020b). La crisis sanitaria deriva en crecimiento (muchas veces exponencial) tanto de personas infectadas como de situaciones críticas y muertes en exceso vinculadas a la pandemia. En este aspecto, la asimetría se manifiesta en las diferentes tasas de contagio y letalidad vinculadas no sólo a los diversos grupos etarios sino también a grupos de población con desigual acceso a cuidados preventivos y terapéuticos.

La crisis económica, por su parte, surge de un doble shock de oferta y demanda que golpeó sobre economías que ya venían inmersas en procesos de estancamiento y problemas de desbalances macroeconómicos y financieros. Sobre estas tendencias se aplicaron políticas de cierre de unidades productivas, centros educativos y trabas de movilidad de personas, las cuales tuvieron un impacto asimétrico sobre la heterogénea estructura productiva de las economías de la región. A las medidas internas, se sumó la caída del comercio internacional para países fuertemente dependientes de la exportación de materias primas (CEPAL, 2020a, CEPAL 2020b, FMI, 2020a, World Bank, 2020).

Estos impactos derivados de la pandemia se descargaron sobre una de las regiones más desiguales del mundo y aceleraron problemas acumulativos (Blofield y Filgueira, 2020), con efectos más intensos en las condiciones de vida de los grupos informales y más vulnerables, quienes sufren pérdidas de ingresos y déficits habitacionales con bajo acceso a servicios públicos básicos, incluyendo los sanitarios. Además, la población más vulnerable no dispone de estabilizadores automáticos de ingresos (i.e. seguro de desempleo) ni acceso a la seguridad social ni ahorros previos, por lo que no sólo su transcurso de la pandemia es más doloroso sino que tendrán mayores dificultades para recuperarse y regresar a sus ya precarias condiciones de vida previas al shock.

Al igual que en otras regiones del mundo, en este contexto crítico se observa un crecimiento de grupos interesados en el ingreso ciudadano o renta básica (RB) como política para la contención de los infortunios de los grupos más desaventajados. El mayor interés es lógico: ante la pérdida masiva de empleo e ingresos cobra relevancia una propuesta que pretende garantizar ingresos de forma universal e incondicional.

Sin embargo, el crecimiento del debate y el apoyo de ciertos grupos a la propuesta de $R B$ en $A L$ no es garantía de que su implementación sea factible en el corto plazo. Para evaluar las posibilidades efectivas de implementación de la RB en los 
países de AL, y sin desconocer los múltiples elementos que en un momento determinado pueden definir la factibilidad de implementación de esta política (De Wispelaere (2016) y Lo Vuolo, 2013b), consideramos aquí tres elementos claves que cobran importancia en el actual contexto de pandemia: i) la naturaleza del vector de riesgo; ii) la estrategia epidemiológica para enfrentarlo; y iii) la profunda vulnerabilidad de la estructura social en la región.

El vector de riesgo se deriva de un virus de alta capacidad de contagio y moderada pero rápida letalidad sin tratamientos claros. Estas características potencian la importancia del acceso a bienes públicos y de la regulación de externalidades, al tiempo que limita (aunque no suprime) la posibilidad de las elites de aislarse. Justamente, para gran parte de la población en AL la falta de adecuado acceso a la salud, saneamiento, viviendas básicas y sistemas de aseguramiento colectivo define una fuerte externalidad negativa que incrementa el riesgo de las medidas como la restricción de movilidad y las imposibilidades de sostener formas mínimas de distanciamiento y confinamiento parcial o total.

En segundo lugar, la estrategia de lockdown y cuarentena aplicada para enfrentar la diseminación de la pandemia hace que, de las cuatro esferas que producen y asignan bienes y servicios (mercado,

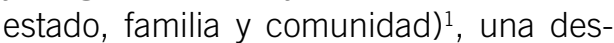
nude sus fuertes limitaciones y las otras

1. Estas cuatro esferas que producen y asignan recursos y coordinan la acción de agentes individuales y colectivos. El imperialismo neoclásico relegó las dos últimas a la irrelevancia y castigó la segunda a una eterna desconfianza, dejando al mercado como el mecanismo natural y preferente para estas funciones. se vean jerarquizadas. Así, se ve seriamente cuestionada la esfera del mercado y su capacidad de resolver eficientemente la asignación de recursos y la coordinación de agentes. En contraste, frente a la estrategia epidemiológica adoptada, se revalorizan: i) el Estado como mecanismo vinculante en la extracción y asignación de recursos y en la coordinación de agentes; ii) las familias como forma básica de producción de aseguramientos y pooling de recursos; iii) la comunidad como espacio potencial de solidaridad y formas no mercantilizadas de intercambio.

En tercer lugar, y con variaciones por país, la pandemia y las políticas adoptadas para enfrentarla no sólo impactan primordialmente en la amplia población más marginada que ya carecía de acceso a condiciones básicas de vida, sino también en grupos muy "vulnerables" a cambios mínimos en su forma de vida. Otros grupos, aun cuando tienen capacidad de respuesta en el corto plazo, muy probablemente no podrán sostenerla si la situación se extiende mucho en el tiempo. Las vulnerabilidades ante shocks externos no son un rasgo coyuntural sino estructural de la región; la magnitud y profundidad del shock pueden ser coyunturales, pero la precariedad de aseguramientos y de acceso a bienes colectivos es estructural en una región que ya mostró y muestra una estructura social muy frágil no sólo frente a shocks externos sino frente a los riesgos vitales propios del ciclo de vida de las personas (enfermedad, vejez, desempleo, etc.).

En este escenario, el desafío que enfrenta la región en materia de ingresos y bienestar frente a la pandemia es triple: elevar a aquellos sectores con déficits extremos antes de la pandemia, evitar la caída de 
sectores afectados por sus efectos y garantizar tasas de reemplazo razonables de ingresos a sectores medios-bajos que sufran pérdidas de empleo y salarios. La propia recuperación económica depende en gran medida de este desafío porque su atención es imprescindible para otorgar certidumbre inter-temporal y evitar un sobre-ajuste negativo del consumo y de la demanda agregada. $Y$ es aquí donde el debate sobre la implementación de la $\mathrm{RB}$ encuentra un escenario si se quiere novedoso o al menos propicio para su consideración como política pública.

Así como a finales del siglo XIX e inicios del siglo XX el descubrimiento de vectores epidemiológicos derivó en una gran inversión en materia de salubridad pública, sería deseable que la pandemia actual derive en sistemas de protección social universales e incondicionales, tanto en la transferencia de ingresos como en el acceso a bienes y servicios públicos colectivos. Esto sería necesario no sólo por razones de derechos o equidad social, sino por razones de eficiencia y eficacia. La RB podría jugar un papel clave en este proceso por sus impactos en el sostenimiento de ingresos de la amplia zona de vulnerabilidad social en la región, financiada con una reforma fiscal progresiva y combinada con el fortalecimiento y amplio acceso universal a bienes y servicios públicos de calidad (Filgueira y Lo Vuolo, 2020). Sin embargo, no hay muchos elementos para predecir que este sea el desenlace.

Aun cuando se registra una creciente retórica favorable a la RB, lo que predomina en la región son estrategias que repiten la naturaleza estratificada, segmentada y excluyente de los sistemas de protección social existentes. Asimismo, no se observan medidas que tiendan a fortalecer los componentes universales de servicios públicos y colectivos claves, y mucho menos proyectos de reforma tributaria que busquen integrar la RB como crédito fiscal de un sistema tributario progresivo.

Pese al fracaso de estas estrategias tanto en la "normalidad" previa como frente a las necesidades derivadas de la crisis de COVID19, en la región continúa un abordaje fragmentado, paliativo, temporalmente acotado y de baja inversión en bienes públicos y de mérito. En contraste, un modelo de universalismo incondicional debería sustentarse en tres pilares que la región nunca ha construido y que siguen sin plantearse en la crítica coyuntura actual: una garantía de ingreso universal, incondicional y permanente integrado con una fiscalidad progresiva estable y una matriz robusta de bienes públicos y de mérito de larga duración.

En las secciones que siguen se analiza la fragmentación histórica de los sistemas de protección social de la región y el rol del mercado laboral como motor de producción de desigualdades y exclusión. Luego se documenta la vulnerabilidad de la estructura social de la región mostrando como los efectos de la pandemia no crean dichas vulnerabilidades sino que agudizan rasgos estructurales de larga data. En este marco se discute la respuesta de los gobiernos ante la pandemia y se señalan sus limitaciones.

Con este escenario de referencia, se concluye que existe una baja probabilidad que los gobiernos adopten programas bajo los principios de la RB, más allá de la retórica reciente. Menos probables es que lo hagan combinando una política de RB con las reformas tributarias necesarias y con el fortalecimiento de los bienes públicos y de mérito, históricamente frágiles en la región. 


\section{Rasgos estructurales de los sistemas de protección social en América Latina}

Los sistemas de protección social en AL se desarrollaron de manera fragmentada, con límites a la expansión de la cobertura y con muy desigual acceso a los beneficios para los distintos grupos de población. En este desarrollo, se observa una evidente tensión entre un discurso universalista de reconocimiento de derechos sociales igualitarios y una práctica particularista que segmenta a la población en grupos gestionados de forma separada y desigual (Lo Vuolo, 2013a).

Con matices por país, en la región nunca se logró replicar las formas de organización económica y social de los Estados de Bienestar maduros, tanto en sus niveles de formalidad salarial, como en los modelos familiares nucleares tradicionales. Pese a no haber completado nunca la industrialización y la primera transición demográfica, el nuevo milenio encontró a buena parte de la región embarcada en procesos propios de la sociedad post-industrial y de la segunda transición demográfica. Estos procesos, combinados con regímenes segmentados y particularista de protección social, amplían la vulnerabilidad social de gran parte de la población tanto en la "normalidad" de la reproducción social como frente a la ocurrencia de shocks externos inesperados.

La elevada economía informal es uno de los elementos que explica estos resultados. No se trata de un problema aislado sino de un particular modo de regulación de la economía y de gestión política. En la región el mercado laboral es un espacio de desigualdad social que se proyecta a otros espacios sociales y al sistema de políticas públicas. Las evidencias históricas permiten afirmar que en AL nunca ha sido posible: i) obtener el ideal de pleno empleo para toda la fuerza laboral; ii) cumplir con los imperativos que el sistema capitalista impone al contrato laboral como mecanismo de asignación de empleos disponibles y distribución equitativa del ingreso; iii) alcanzar una cobertura universal e igualitaria con las políticas de seguro social.

Pese a ello, las instituciones sociales continúan organizándose con reglas que buscan preservar la primacía distributiva del mercado laboral y que tratan a la pobreza y la exclusión social como una cuestión residual y hasta transitoria. Por el contrario, para una enorme masa de población, la pobreza y las carencias de bienestar no son derivadas de pérdidas circunstanciales de ingresos por crisis cíclicas o reconversiones productivas, sino problemas estructurales resultantes del modo de funcionamiento tanto del mercado laboral como de las instituciones de seguridad social.

\section{2.l Los sistemas de seguridad social contributiva: exclusión, estratificación y sostenibilidad}

Los mercados laborales de la región están segmentados por productividad, tamaño y capacidad regulatoria del Estado, por lo que limitan seriamente la cobertura de los seguros sociales excluyendo a los grupos más vulnerables de la población. Así, los sistemas de seguro social contributivos en la región registran, en general, tres rasgos distintivos: déficit de cobertura, marcada estratificación y déficits de sostenibilidad demográfica y fiscal.

En la práctica, los seguros sociales se encuentran estratificados por niveles de 
ingresos y categoría laboral; así, los grupos de población que más requieren de aseguramiento social son quienes menos acceden y, en caso de acceder, lo hacen en condiciones más precarias. Por ejemplo, las tasas de reemplazo de jubilaciones y pensiones contributivas suelen favorecer a los grupos de altos ingresos y a los empleados públicos, pero son muy bajas para trabajadores independientes, rurales o domésticos. Además, amplios grupos de población no tienen cobertura ni de pensiones, ni de desempleo, ni de licencias y enfermedades.

Sin embargo, pese a que cubren sólo a una parte de la población, los seguros sociales contributivos requieren de subsidios desde rentas generales por sus bajas tasas de sostenimiento y crecientes déficits financieros. En otras palabras, los subsidios para sostener los déficits de estas instituciones profundizan la desigualdad regresiva derivada de la cobertura. Por ejemplo, cuando $20 \%$ de la población más pobre no tiene cobertura de los seguros sociales en $\mathrm{AL}$, los déficits de estas instituciones de seguro social se financian en muchos casos con trasferencias de rentas generales cuya recaudación proviene principalmente de impuestos regresivos como el IVA. Además, los propios aportes a la seguridad social son raramente progresivos.

\subsection{Los programas de transferencias de ingresos no contributivos en América Latina: insuficiencia, focalización restringida y transitoriedad}

Para sostener los ingresos de los grupos más vulnerables, en el escenario previa- mente sintetizado, se han diseminado múltiples programas de transferencias condicionadas de ingresos (PTC) que buscan asistir a algunos grupos que no están cubiertos por los seguros sociales en la región. Estos programas se piensan como compensatorios de la carencia de recursos y suelen tener como unidad receptora del beneficio al grupo familiar (Cecchini y Atuesta, 2017). La focalización y las condicionalidades que caracterizan a estos programas pretenden "no dar dinero a quien no lo necesita", en tanto se considera que en caso contrario se afectarían los incentivos para la oferta laboral en el mercado de empleo.

Estos programas, si bien ayudan a los sectores más desaventajados, son débiles para promover la salida desde la situación de pobreza y para construir canales de movilidad social ${ }^{2}$. Su propia concepción relega dos funciones centrales de los sistemas de protección social: 1) su rol preventivo y asegurador frente a contingencias que afectan al conjunto de la población: 2) su rol integrador de partes desiguales en instituciones igualitarias. Así, los PPT no resuelven problemas de cobertura, no promueven movilidad social y no remueven la segmentación desigual de la política pública.

En relación con la RB, los PTC demuestran que no puede garantizarse un ingreso básico permanente que sirva para prevenir insuficiencia de ingresos con tecnologías focalizadas por más amplia

2. Un caso particular es la expansión en las últimas décadas de programas de pensiones no contributivas o semi-contributivas, que mejoraron sustantivamente la cobertura de los adultos mayores, aunque con diferencias entre países según las diversas trayectorias institucionales y tradiciones políticas. Ver Arza (2013 y 2019). 
que sea la cobertura. Tampoco los PTC pueden garantizar la efectiva consagración de los derechos sociales establecidos formalmente en las constituciones y leyes de los países de la región. Los derechos sociales son derechos colectivos que, en gran medida, se hacen efectivos habilitando el acceso al consumo de bienes y servicios (medicamentos, servicios, transferencias de dinero, etc.). Por ser derechos colectivos no pueden hacerse efectivos con programas segmentados según características particulares de cada persona o grupos.

Cuando los riesgos sociales se individualizan y se pretende aplicar un programa para cada caso particular, o directamente derivar al seguro privado individual la cobertura, se abre la puerta para que el Estado se desligue de su responsabilidad sobre los riesgos sociales de la población, transfiriendo el problema al mercado, grupo familiar, comunidad, etc. Por el contrario, los derechos sociales son derechos colectivos sobre los recursos comunes de la sociedad y quienes deberían tener el poder para reclamarlos al Estado son las personas. Justamente, es la universalidad incondicional de programas como la RB lo que garantiza que los más vulnerables estén colectivamente representados e integrados a los derechos que gozan los grupos más favorecidos de la población.

De aquí puede entenderse el motivo por el cual es difícil considerar a los PPT como un primer paso de un camino hacia la implementación de la RB en los países latinoamericanos (Lo Vuolo, 2012). Las reglas operativas de los PPT son contrarias a los principios que guían a la RB. La RB no es cualquier política de transferencias de ingreso y los PPT sirven más bien para distorsionar y desplazar el debate creando la falsa imagen de que "ya existe algo similar" y no hay que innovar.
Lo anterior también ilustra un error recurrente: comparar a la RB como un programa alternativo en el estrecho campo de la gestión de programas asistenciales. La RB no es un programa asistencial sino una política transformadora de las relaciones fundamentales que organizan el mercado de empleo y el sistema de protección social. Pretende cambiar tanto la lógica de funcionamiento de los seguros sociales como de los programas asistenciales, abandonando la estructura fragmentada, condicionada y desigual del actual sistema para reconstruirlo en base a políticas universales, incondicionales y promotoras de igualdad.

¿Qué se ha modificado con la crisis derivada de la propagación de la pandemia del COVID-19? Lamentablemente, no mucho. Pese a la profundización del déficit de bienestar de gran parte de la población y a la ampliación de la vulnerabilidad social, los programas aplicados en la emergencia de la pandemia se identifican con las tradiciones y estructuras de las políticas públicas ya existentes en la región.

\section{Vulnerabilidad social antes y durante la Pandemia}

Para que se entienda el contexto del debate, es ilustrativo recordar algunos indicadores de la situación económica y social en AL. En los primeros 14 años del inicio de siglo, con variantes por país, la región registró un proceso de reducción de la pobreza y de la pobreza extrema; sin embargo, a partir de 2015 dicho proceso se estancó y empezó a revertirse en 2018 y 2019. Al inicio de la pandemia de COVID19, 25\% de la población se encontraba en situación de muy alta vulnerabilidad a la pobreza, con ingresos entre 1 y 1,8 


\section{Porcentaje de la población en estratos bajos de ingreso per-cápita del hogar. Estimaciones para el 2019}

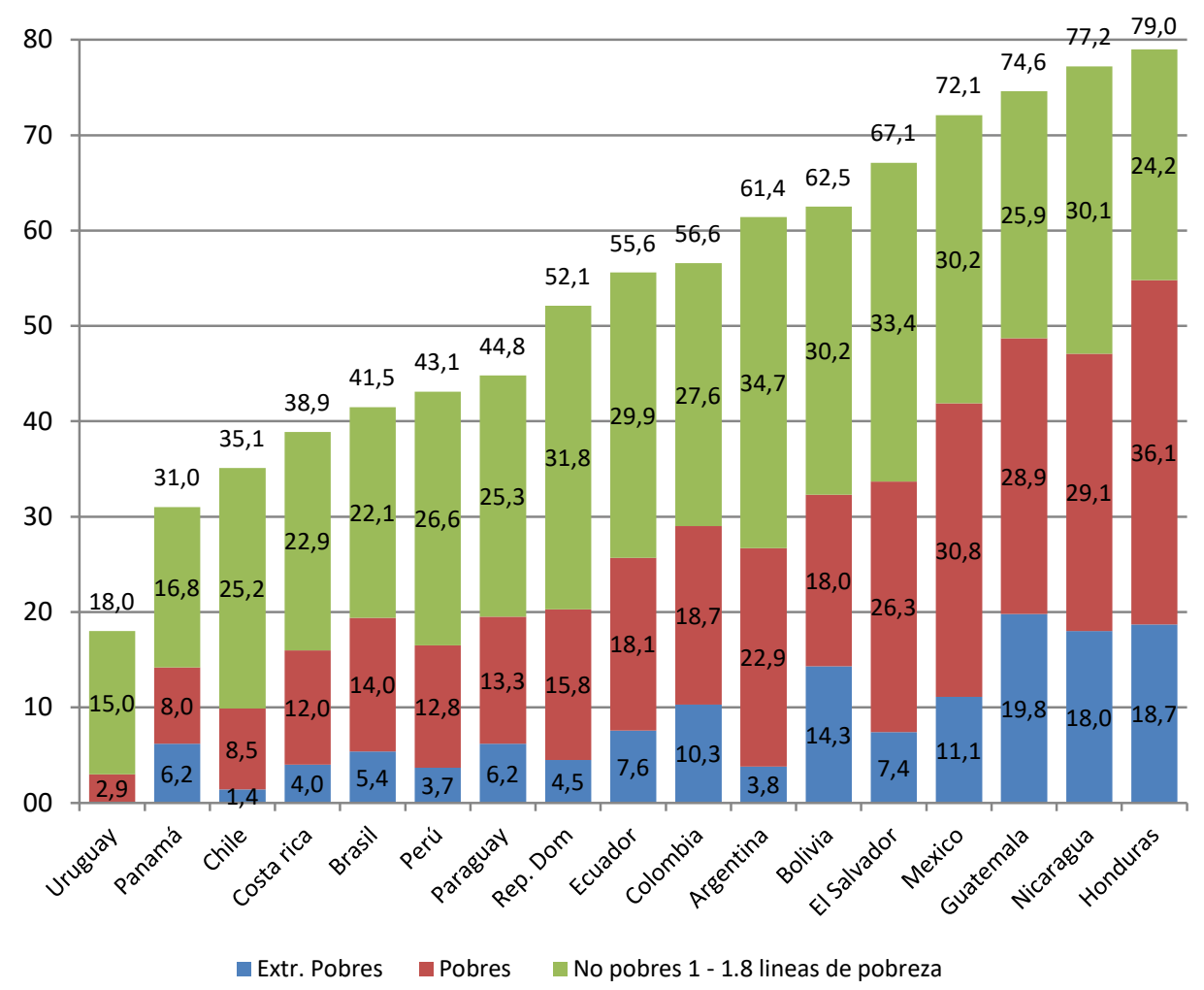

Fuente: CEPAL en base a tabulaciones especiales de las Encuestas de Consumos de los Hogares.

líneas de pobreza. Cuando se considera en su conjunto a la extrema pobreza, la pobreza y los estratos bajos no pobres, un solo país de la región registraba un valor por debajo de $20 \%$ de la población; la mayor parte presentaba valores por encima de $50 \%$ de las personas en las tres categorías mencionadas (ver gráfico).

A la insuficiencia de ingresos corrientes se suma la carencia de ahorros de los grupos más empobrecidos, lo cual amplía las desigualdades frente al shock de la pandemia y las políticas de confinamiento. Se estima que en más del $30 \%$ de los hogares y en algunos casos en más de la mitad, la capacidad de ahorro es negativa o es un por- centaje menor al $10 \%$ de sus ingresos corrientes (Blofield y Filgueira, 2020). Por lo tanto, los impactos de la pandemia no sólo son desiguales según el tipo de empleo y el nivel de ingresos, sino también existe un diferencial muy grande en el ahorro y stock de capital acumulado previamente (incluyendo la posibilidad de acceso al crédito y de capital social). Son estos stocks los que, sumados al efecto sobre el flujo, permiten sostener niveles de consumo adecuados ante un shock de las magnitudes que estamos considerando.

Además, en la región persisten déficits habitacionales que ya generaban riesgos sanitarios previamente al shock de la 
pandemia y que se agudizan ante un uso más intensivo de la infraestructura hogareña por el confinamiento. Además, existe una amplia proporción de población que dada la caída de sus ingresos tiene serias dificultades para pagar alquileres y servicios básicos, obligando incluso a ajustar el gasto en otros rubros esenciales por el riesgo de perder directamente el acceso a la habitación o al servicio.

Asimismo, como ya se señaló, la informalidad o ausencia de cobertura de seguridad social se encuentra fuertemente concentrada en los quintiles más pobres, en las mujeres, en los sectores rurales, y la población indígena y afrodescendiente (CEPAL, 2018). El 75\% de los hogares en el menor quintil de ingresos posee niñas, niños y adolescentes menores de 18 años, al tiempo que los hogares biparentales representan el $52 \%$ y los monoparentales el $20 \%$ en este mismo quintil. Estos indicadores contrastan con el quintil más alto de la distribución de ingresos donde el 68\% de los hogares no tienen hijos y/o hijas, y solo el $6 \%$ son hogares monoparentales.

En otras palabras, la mayoría de los hogares en los quintiles más bajos están integrados por niñas, niños o adolescentes que con el cierre de escuelas están confinados en su hogar y requieren cuidados y comidas que muchos recibían previamente vía programas de comidas escolares gratuitas o subsidiadas. Estos hogares se ven especialmente afectados por los efectos económicos de las políticas aplicadas para enfrentar la pandemia. Asó, una encuesta del BID reportó que $69 \%$ de las personas en hogares de bajos ingresos declaran haber perdido su trabajo debido a la pandemia, en comparación con el $26 \%$ en los hogares de mayores ingresos (Bottan, Hoffmann y VeraCossio, 2020). Según datos de la misma encuesta, el primer quintil de ingresos también reporta inseguridad alimentaria generalizada.

Los efectos económicos desparejos también interactúan con la desigualdad de género dentro del hogar. Los datos de uso del tiempo previos a la pandemia muestran una brecha de género significativa en tareas del hogar y de cuidado en AL: la diferencia en la cantidad de horas dedicadas de mujeres y de varones es de 18.1 horas por semana. Según datos de la OCDE, esta cifra es notablemente superior a la brecha en el sur de Europa (10.9), los países anglosajones (5.9) y los países nórdicos (3.5). Este promedio oculta la variación entre mujeres según ingreso.

Como se sabe, los hombres trabajan poco en el hogar y no cambian su comportamiento durante su ciclo de vida mientras que el trabajo no remunerado de las mujeres se incrementa sustancialmente durante sus años de maternidad. Pero además, las mujeres de bajos ingresos realizan más trabajo no remunerado que las de altos ingresos (debido a una más temprana y mayor fertilidad y a la falta de capacidad para subcontratar), y esta carga desigual es extrema en las familias monoparentales, de las cuales la gran mayoría son monomaternales. En estos hogares, las madres proporcionan tanto ingresos como cuidados, con poco apoyo de los padres no residentes (Cuesta, Hakovirta y Jokela, 2018). Todas estas desigualdades se incrementaron con el shock de la pandemia.

Para terminar de exponer el escenario social que se registraba en la región antes del azote de la pandemia y las políticas aplicadas para mitigar sus efectos, vale una mención sobre la desigual estructura de gastos de consumo. En términos generales, 
la mayor parte del gasto en cada uno de los rubros es realizada por los dos quintiles de ingreso más altos, como reflejo de la elevada concentración del ingreso en AL.

Cuando se suma el gasto en alimentación y vivienda el quintil más pobre y en menor medida el siguiente destinan siempre más de $40 \%$, y en algunos casos más de $60 \%$ del total de sus gastos a dichos rubros. El otro rubro que sigue en importancia es el transporte. En la región se observa un aumento de las participaciones de gasto en educación, en salud y en transporte conforme aumenta el ingreso.

En otras palabras, con el aumento de ingresos los hogares utilizan, en menor medida, la educación pública, la salud pública y el transporte público y migran a la educación privada, la salud privada y el transporte privado. El acceso a la salud, aún en los sectores pobres, en muchos casos requiere de un fuerte componente del gasto de bolsillo. Esto configura un patrón de consumo y un estilo de desarrollo segmentado y muy difícil de sostener en el tiempo que afecta mayormente a los grupos más vulnerables.

\section{La pandemia y las estrategias de mitigación ${ }^{3}$}

Lo anterior es suficiente para advertir sobre los impactos brutales que la propagación de la pandemia y las estrategias aplicadas para su contención han tenido sobre los grupos más subordinados de AL. También advierte que, de prolongarse los confinamientos y lock-down como forma de combatir la pandemia, las tendencias señaladas seguramente empeorarán con

3. Este apartado tiene como fuentes principales a Filgueira, Galindo, Giambruno y Blofield (2020) y Blofield, Giambruno y Filgueira (2020). impactos tremendos sobre la población en situación de pobreza -y más aún en extrema pobreza-.

Considerando que la mayor parte de los países de la región conocieron sus primeros casos confirmados de COVID19 entre finales de febrero y la primera quincena de marzo de 2020, al 31 de agosto de 2020 podían distinguirse tres grupos de países.

Un primer grupo presentaba una cantidad de casos por 100 mil habitantes y muertes por millón habitantes modestos: Honduras, Guatemala, El Salvador, Uruguay, Costa Rica y Paraguay. De este grupo, solamente Uruguay presentaba, además de las magnitudes más bajas de la región, una forma de la curva que indica logros importantes en la contención, mientras que Paraguay ya empezaba a mostrar tendencias preocupantes y el resto ya había adquirido la forma exponencial4.

El segundo grupo, que incluye a Argentina, Bolivia, Colombia y República Dominicana, presentaba valores intermedios en casos por 100 mil y muertes por millón de habitantes. Aquí se observan curvas exponenciales o que tienden a tal forma funcional en ambos indicadores.

La peor configuración la registraba un tercer grupo que superaba largamente al resto en casos por 100 mil y que con variantes presentaba un comportamiento de los óbitos por COVID19 también preocupante. Chile y Perú presentaban por lejos la mayor presencia de casos detectados, aunque eran los países con más test percápita realizados en toda la región. Por ello en parte, las muertes, si bien presentan un

4. Es importante destacar que, en tanto Uruguay, Costa Rica, Paraguay, y El Salvador presentaban niveles de testeo por cada mil habitantes moderados a altos en el contexto regional; ello no es cierto en Guatemala y Honduras. 
comportamiento exponencial, lo hacen a distancia importante de la misma pauta de los casos acumulados. México, y en menor medida Ecuador, aparecen como los más extremos ya que las muertes por millón de habitantes presentan un comportamiento similar al de los casos por 100 mil habitantes. Ello sugiere una alta tasa de letalidad, posiblemente influenciada por una baja captación de los casos reales.

En este contexto, en casi todos los países los gobiernos -con contadas excepciones- se movieron en forma relativamente rápida a estrategias que combinaban al menos cuatro medidas profundamente disruptivas de la vida económica y social: i) cierre de fronteras; ii) cierre de escuelas y centros educativos; iii) cierre de empresas consideradas no esenciales (y disminución de las actividades aún de servicios y empresas esenciales como el transporte público); iv) cuarentenas en el hogar obligatorias para toda la población, o voluntarias (con fuertes exhortaciones) con cuarentenas obligatorias para la población en riesgo sanitario o contagiada.

Sin embargo, el inicio de las medidas económicas y sociales casi siempre presentó un "lag" respecto a las de contención epidemiológica. Esto contrasta con el comportamiento de la respuesta social en algunos países centrales, especialmente en Europa. En estos países con fuertes sistemas de protección social y Estados de Bienestar consolidados, los programas de la seguridad social funcionaron como estabilizadores automáticos en materia económica y social. En particular los seguros de desempleo y enfermedad se activaron en el mismo momento en que se generó la disrupción epidemiológica y proporcionaron ciertas certezas en el contexto de incertidumbre generada por las dinámicas de lock-down y confinamiento.
En cualquier caso, también el panorama fue heterogéneo en AL. Los casos extremos de México y República Dominicana muestran un gran desfasaje entre las medidas epidemiológicas y las económicas y sociales, mientras que en Uruguay, debido a sus estabilizadores automáticos (seguro de desempleo) y a su más robusto sistema de protección social no contributiva, hubo una respuesta temprana de mitigación social. Esto explica el éxito uruguayo en su exhortación a la población para una "cuarentena voluntaria", así como la disminución en las exigencias epidemiológicas que permitió un retorno a la actividad social y económica mientras se mantuvieron y hasta incrementaron las acciones de mitigación social.

Entre estos extremos, si bien las estrategias de los gobiernos para enfrentar los efectos económicos y sociales de las medidas de contención epidemiológicas han sido muy variadas, pueden identificarse ciertas acciones comunes dirigidas a las familias y empresas.

Por un lado, están las acciones dirigidas a las familias. En este segmento, tres son acciones directas de transferencias monetarias o distribución directa de alimentos $\mathrm{u}$ otros bienes a los hogares, incluyendo aquellas tendientes a evitar situaciones de extrema vulnerabilidad (población de calle) o de necesidad de movilidad de quienes son parte de la población con alto riesgo clínico (adultos mayores). Otras dos son: i) exoneraciones, condonaciones o suspensiones de eventuales cortes de servicios públicos por falta de pago, así como de garantías de acceso a vivienda o habitación cuando no se tiene su propiedad; ii) un conjunto de exoneraciones, subsidios y créditos orientados a empresas y que procuran sostener la actividad y el empleo.

La siguiente figura ilustra estas acciones. 


\section{Accionar estatal en materia de Protección Social}

\section{Acciones de protección social}
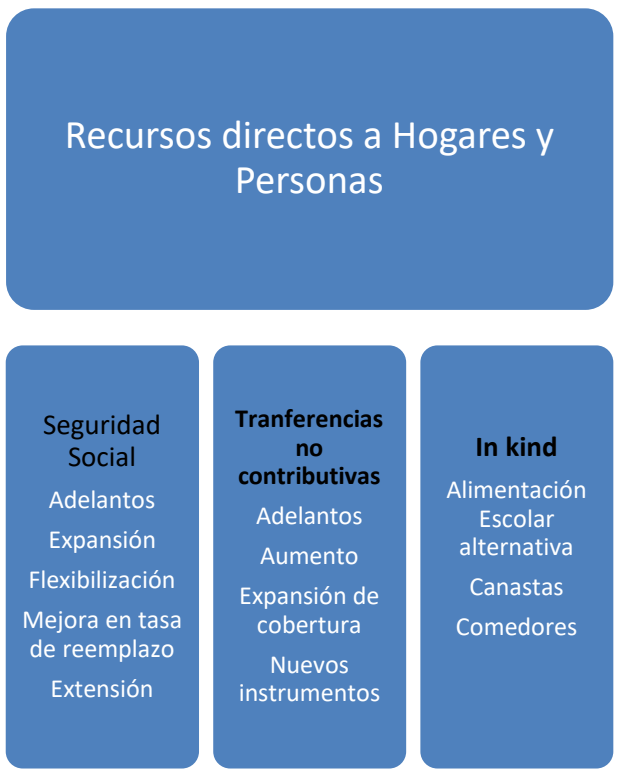
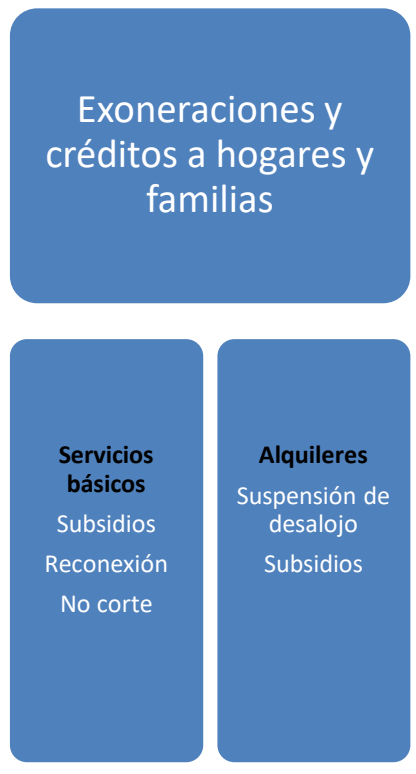
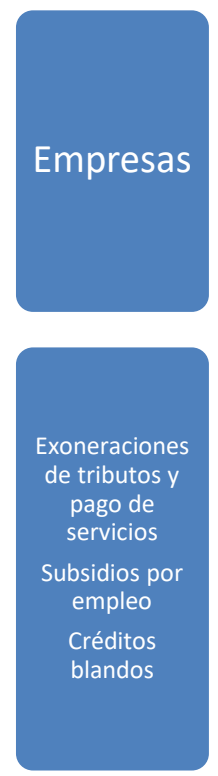

Fuente: Filgueira, Galindo, Giambruno y Blofield (2020).

En lo que refiere particularmente a las transferencias monetarias y transferencias en especies, algunos países optaron por fortalecer los programas asistenciales focalizados ya existentes incrementando sus beneficios con pagos extraordinarios. Un ejemplo de esto es el caso de Argentina, que realizó una pago extraordinario a beneficiarios de distintos programas tales como Asignación Universal por Hijo (AUH) y su complemento la Asignación Universal por Embarazo (AUE); también a beneficiarios de pensiones no contributivas por vejez, entre otros. En el mismo sentido, en Colombia se otorgaron tres giros adicionales a los beneficiarios del programa Familias en Acción y Jóvenes en Acción, mientras que en Uruguay se duplicaron los montos tanto de la Tarjeta Uruguay Social, como de las Asignaciones Familiares - Plan de Equidad. Por otra parte, otros países han optado por aumentar la cobertura poblacional de los programas existentes, tal es el caso de Brasil con el programa Bolsa de Familia, Guatemala con el programa de aporte económico al adulto mayor, y México para el programa Sembrando Vida.

Otros gobiernos optaron por adelantar pagos, principalmente para pensiones no contributivas por vejez. Tal es el caso del Programa Juntos y Pensión de Perú, el anticipo del decimotercer sueldo a pensionados y jubilados en Brasil, el adelanto 
de 4 meses del Programa Pensión para el Bienestar a adultos mayores y personas con discapacidad en México, el anticipo de la Pensión Alimentaria Para Adultos Mayores en Situación de Pobreza en Paraguay, así como del Régimen No Contributivo y del Seguro de Invalidez, Vejez y Muerte en Costa Rica.

Adicionalmente, en muchos países se crearon nuevas prestaciones focalizadas, que combinan la incorporación de nuevos beneficios a la población ya beneficiaria de otros programas y la incorporación de nueva población objetivo. Tal es el caso del Ingreso Familiar de Emergencia en Argentina, el Programa de Seguridad Alimentaria "Ñangareko" en Paraguay, los bonos "Yo me quedo en casa" y Bono Universal Familiar en Perú, el Bono Universal y Bono Familia en Bolivia, el Auxilio emergencial do Governo Federal en Brasil y el Bono de Emergencia COVID-19, Ingreso Familiar de Emergencia e IFE 2.0 en Chile.

En lo que refiere a servicios públicos, las medidas van desde el subsidio de los pagos para hogares de bajos ingresos, la postergación de los pagos sin intereses, y la prohibición de corte de servicios por falta de pagos. Las políticas de subsidio implementadas se focalizan en la población más vulnerable, mientras que el diferimiento de pagos sin intereses, y la prohibición de corte de servicios por falta de pago suele ser universal. Medidas como la prohibición de aumentos en los precios de alquileres y suspensión de pagos han sido tomadas en una menor cantidad de países.

Si bien la mayor parte de los países aplicó medidas de protección al empleo y/o al salario de los trabajadores, las diferencias por países son sustanciales, considerando que sólo siete países de la región cuentan con seguro de desempleo que en la mayoría de los casos es de muy baja cobertura: Argentina, Uruguay, Chile, Brasil, Ecuador, Colombia y Venezuela. Uruguay implementó un seguro de paro flexible y parcial. Se aplicaron subsidio al ingreso laboral (Argentina, Brasil, Chile, Guatemala, entre otros) y en algunos casos el pago se realiza directamente a las personas, mientras que en otros se realiza a través del empleador. Sólo México y Argentina optaron por la prohibición de despidos, pero no existen indicadores acerca de su efectividad.

Más allá de las magnitudes de gasto y esfuerzos de gestión que implican estas acciones, se observan problemas que se trasladan a déficits en cuatro parámetros básicos: i) timing (lentas respecto al confinamiento); ii) cobertura (fragmentadas e incompletas); iii) suficiencia (valores marginales de transferencias); y iv) lapso de tiempo (acotadas a la emergencia aguda sin considerar duración más larga y efectos inter-temporales). Estos déficits son el resultado lógico de las señaladas características de los modelos de protección social de la región: paliativos ante el daño antes que preventivos, con déficits severos de cobertura, con esfuerzos fiscales insuficientes y acotados en el tiempo. A lo anterior se agregan los problemas estructurales en la provisión de bienes públicos y bienes colectivos (infraestructura urbana acceso a servicios públicos básicos, saneamiento, acceso a la salud, seguridad) que obviamente no pueden resolverse en el corto plazo y que muestran sus debilidades frente a la emergencia sanitaria y las estrategias de confinamiento.

En síntesis, la respuesta regional frente a la pandemia fue importante pero reproduciendo las características distintivas de sistemas de protección social fallidos y sistemas de provisión de bienes públicos 
frágiles. Si bien el shock es muy agudo, no creó la vulnerabilidad social generalizada sino que desnudó los déficits estructurales y sus consecuencias más agudas. También, es evidente que las políticas aplicadas para paliar sus efectos siguen la misma matriz fallida e inarmónica que hace tiempo caracteriza a los sistemas de protección social en la región.

En general, la clase política parece considerar al COVID19 como un shock exógeno agudo que tenderá a desaparecer y al cual se puede culpar no sólo de los problemas actuales sino de los de larga data. También que lo que se necesita es fortalecer temporalmente los programas existentes, ampliando coberturas y beneficios pero en ningún caso se observa un avance hacia políticas alternativas como la RB ni tampoco el diseño de programas que apunten a paliar los déficits de infraestructura social, incluyen los que afectan al propio sector de salud.

\section{Posibilidad o espejismo para la RB y los bienes públicos en la agenda regional}

El análisis previo pretende describir, si bien de forma sucinta, el escenario en el cual se debate la propuesta de la RB en AL. Aun cuando es evidente que la propuesta tiene en la región muchos motivos para afianzarse, lo que se observa es la presencia una vez más de bloqueos estructurales que traban tanto la adopción de la RB como de políticas complementarias y consistentes con la misma. Más bien, lo que se observa es una continuidad de la matriz que combina seguro social para el empleo formal y políticas asistenciales focalizadas para grupos seleccionados, que se han potenciado de forma temporal durante la pandemia. Además se ha potenciado la idea de que se necesitan programas para una emergencia que se considera temporal y no para prevenir shocks y cambiar el modo de funcionamiento del sistema en su conjunto.

Por supuesto que siempre puede esperarse que las elites y los actores políticos con peso aprendan de esta experiencia. En ese caso, las posibilidades de la RB en la región dependen más bien de que las tres crisis combinadas y asimétricas, sirvan para colocar en la agenda pública la necesidad de superar tanto del déficit de bienes públicos y de mérito como los límites de los seguros sociales y de las políticas de transferencias monetarias focalizadas y condicionadas. En otras palabras, la implementación de una RB que alcance los objetivos pretendidos depende de que se acepte revisar el conjunto de la arquitectura de los regímenes de protección social en la región, tanto de su base fiscal como de las instituciones que proveen bienes y servicios colectivos y transferencias monetarias contributivas y no contributivas.

Parte de la explicación de los bloqueos está en las herencias institucionales de estos regímenes, como así también en las percepciones morales de la ciudadanía, en general, y de las élites con peso político en particular. En la región son fuertes las preferencias por estructuras institucionales basadas en las posiciones desiguales en el mercado laboral, las condicionalidades punitivas que exigen contrapartidas a las personas, la separación institucional entre políticas contributivas y no contributivas, etc.

La reticencia de los partidos políticos a propuestas como la RB también está per- 
meada por estas preferencias. En general, se observa que aquellas políticas con mayor espacio para el ejercicio del control político sobre las acciones de las personas, son las que más logran consenso entre la clase política y la clase dirigente en general (Lo Vuolo, 2015). Y esto también se ha consolidado con la pandemia, en tanto se han multiplicado las bases de datos y los instrumentos que registran y controlan las condiciones de vida de las personas con la "excusa" del seguimiento por motivos de salud pública.

En el caso de los sindicatos la propuesta no termina de ser digerida por una tradición corporativa y proclive a sostener la fragmentación institucional que emerge del mercado laboral. La idea es que el derecho a un ingreso universal e incondicional puede erosionar la estructura construida sobre los derechos laborales, que el carácter individual del beneficio lleva a erosionar la representación colectiva y la intermediación sindical, así como que no es conveniente la igualación de derechos entre trabajadores formales e informales.

En el movimiento de mujeres también se observan apoyos y reparos a la propuesta de la RB (Rodríguez Enríquez, 2013). Por una parte, se reconoce que esta política debería mejorar el bienestar y fortalecer la autonomía de las mujeres, sobre todo en comparación con los actuales programas que las tiene como operadoras intermediarias en un contexto paternalista. Pero sus impactos sobre las alternativas laborales de las mujeres son observados con cautela. Por un lado, se entiende que debería ser una mejor plataforma para la inserción laboral femenina, facilitando una distribución más equitativa y racional de los tiempos de trabajo entre hombres y mujeres. Pero al mismo tiempo, surgen dudas porque, para que esto suceda, son necesarias acciones complementarias en materia laboral y de distribución del trabajo no remunerado que no se consideran factibles.

Lo hasta aquí expuesto señala que puede ser un error promover la aplicación de una RB como una política aislada y atada a la emergencia, como a veces parece ser el objetivo inmediato de muchos grupos de activistas que promocionan la propuesta en la región y en el mundo. Junto con la promoción de la RB hay que promover otros cambios para reconstruir los débiles y desiguales sistemas de protección social en la región. Esto involucra cambios por el lado del gasto como también de los recursos y en la prestación de servicios públicos colectivos.

Por ejemplo, la RB debería promoverse junto con sistemas nacionales de cuidado que garanticen descargar las tareas no remuneradas que hoy recaen principalmente en las mujeres y que se han potenciado con la pandemia. Esto además ayudaría a generar empleos en áreas que hoy no están desarrolladas. Lo mismo puede decirse de la inversión y mejora de calidad en bienes y servicios colectivos y de mérito que registran serios déficits en la región.

También, para tener impactos positivos en el mercado de empleo, la RB debería acompañarse con políticas que tiendan a reducir las horas de trabajo en el empleo, a redistribuir las horas de trabajo no remuneradas y a mejorar la formación de la fuerza de trabajo. Lo anterior debería complementarse con una reforma tributaria que revierta el sesgo regresivo de los actuales sistemas y que incorpore una serie de tributos consistentes con la propuesta que no existen o tienen bajo peso en la región (impuestos a la herencia, a las grandes fortunas, a los daños ecológicos, a las rentas financieras, etc.). 
Asimismo, la RB debería promocionarse como forma de revitalizar a las alicaídas democracias de la región. No debe extrañar que la crisis del sistema representativo que se observa en muchos países de $A L$ se verifique en sociedades que no facilitan la integración social y mucho menos la movilidad social. La necesidad de integrar instituciones igualitarias también abarca a las instituciones políticas de la democracia "real"; su actual funcionamiento no privilegia la participación de la ciudadanía y la necesaria autonomía personal que la misma requiere. En la organización política de los países de la región no está incorporada la idea de que la independencia política de las personas requiere independencia económica. Por el contrario, el sistema funciona con fuerte concentración de poder, escasa auditoría ciudadana y fuerte dependencia de la vida de las personas de los vaivenes del sistema político.

La posibilidad de ejercer autónomamente los derechos políticos y sociales depende del acceso a recursos sin generar dependencia y esto solo lo puede garantizar con un sistema institucional que apunte a distribuir recursos de forma universal e incondicional. Hoy lo que prima en la región es el paternalismo estatal, al corporativismo fragmentado y al clientelismo político en el sistema de políticas sociales para lo cual son funcionales los actuales programas de transferencias focalizadas y condicionadas de ingresos que seleccionan personas entre los grupos necesitados. En términos generales, las democracias latinoamericanas, incluso allí donde se observan mejoras en indicadores de bienestar de los grupos más postergados de la población, siguen funcionando bajo concepciones y con políticas públicas que concentran el poder y el control social que traban el avance de la propuesta de la RB.
En fin, AL registra un escenario que justifica y hasta reclama la pronta implementación de una política de RB pero difícil que avance si se promueve su aplicación en forma aislada, sino que se debe promover la RB como parte de un paquete de políticas que cambien la actual dinámica distributiva. Una política como la RB obligaría a cambios en muchos elementos político-institucionales, especialmente aquellos que revaloricen la importancia de la promoción de un proyecto emancipador de las personas.

La RB no es un programa más entre tantos programas sociales que pululan en la región, sino un elemento clave de un nuevo diseño institucional que sea capaz de afrontar no sólo shocks como los de la pandemia, sino también los desafíos más estructurales de los sistemas económicos, políticos y sociales de la región. Sus méritos son justamente los que alimentan los propios bloqueos derivados de la herencia institucional y de la poca predisposición de las elites a modificar la dinámica de los sistemas sociales en la región.

\section{Bibliografía}

Arza, C. (2019). 'Basic old age protection in Latin America: Non-contributory pensions, coverage expansion strategies and ageing patterns across countries', Population and Development Review, Vol. 45, $\mathrm{N}^{\circ} \mathrm{S} 1, \mathrm{pp} .23-45$.

Arza, C. (2013). 'Basic pensions in Latin America: Towards a rights-based policy?', en Lo Vuolo, R. (Ed.), Citizen's Income and Welfare Regimes in Latin America. From Cash Transfers to Rights. New York, Palgrave-McMillan.

Blofield, M. y Filgueira, F. (2020). COVID-19 and Latin America. Buenos Aires, Ed. Cippec. 
Blofield, M, Giambruno, C. y Filgueira, F. (2020). Policy expansion in compressed time: Assessing the speed, breadth and sufficiency of post-COVID-19 social protection measures in 10 Latin American countries. Santiago de Chile, CEPAL.

Bottan, N., Hoffmann, B. y Vera-Cossio, D. (2020). Coronavirus Survey Results Show Big Impacts, Linkages between Labor Markets and Inequality. Ideas Matter Blog. Inter-American Development Bank, May 8.

Cecchinl, S. y Atuesta, B. (2017). Programas de transferencias condicionadas en América Latina y el Caribe: tendencias de cobertura e inversión. Santiago de Chile, CEPAL.

CEPAL (2020a): https://www.cepal.org/es/ publicaciones/45445-dimensionar-efectos-covid-19-pensar-la-reactivacion. Santiago de Chile, CEPAL.

CEPAL (2020b): El desafío social en tiempos del COVID-19. Informe Especial COVID-19, No.3. Santiago de Chile, CEPAL.

CEPAL (2018): Panorama social de América Latina, 2017. Santiago de Chile, CEPAL.

Cuesta, L., Hakovirta, M. y Jokela, M. (2018). "The antipoverty effectiveness of child support: Empirical evidence for Latin American countries". Social Policy Administration, 52, pp. 1233-1251.

De Wispelaere, J. (2016). "Basic Income in Our Time: Improving Political Prospects Through Policy Learning?", Journal of Social Policy, Vol. 45, 4. October, pp. 617634

Filgueira, F. y Lo Vuolo, R. (2020). La reducción del espacio público en América Latina. Elementos para la construcción de una contra-hegemonía en la producción de bienes y servicios sociales. Documentos de Trabajo Ciepp N 105. Buenos Aires, Ciepp.
Filgueira, F., Galindo, L., Giambruno, C. y Blofield, M. (2020). América Latina ante la crisis del COVID-19: vulnerabilidad socioeconómica y respuesta social. Santiago de Chile, Documento CEPAL, en prensa.

FMI (2020a). https://blogs.imf.

org/2020/06/26/outlook-for-latin-americaand-the-caribbean-an-intensifying-pandemic/. Washington, FMI.

FMI(2020b). https://www.imf.org/external/datamapper/NGDP_RPCH@WEO/ OEMDC/ADVEC/WEOWORLD. Washington, FMI.

Lo Vuolo, R. (2015). "The limits of autonomy in Latin American social policies: promoting human capital or social control?", European Journal of Social Theory, special issue on "Modernity and capitalism", Casassas, D. y Wagner. P. (ed.), vol. 19, no. 2, 2015.

Lo Vuolo, R. (2013a). "Introduction". En Lo Vuolo, R. (ed.), Citizen's Income and Welfare Regimes in Latin America. From cash transfers to rights. New York, Palgrave-McMillan.

Lo Vuolo, R. (2013b). "Epilogue". En Lo Vuolo, R. (ed.), Citizen's Income and Welfare Regimes in Latin America. From cash transfers to rights. New York, Palgrave-McMillan.

Lo Vuolo, R. (2012). "Prospects and Challenges for the Basic Income Proposal in Latin America", Basic Income Studies, Volume 7, Issue 1, June.

Rodríguez Enríquez, C. (2013). "Should Citizen's Income Become a Goal for Feminism in Latin America?". En Lo Vuolo, R. (ed.), Citizen's Income and Welfare Regimes in Latin America. New York, Palgrave McMillan.

World Bank (2020): https://www.bancomundial.org/es/publication/global-economic-prospects. Washington, World Bank. 
